\title{
Norois
}

Environnement, aménagement, société

206 | 2008/1

Itinéraires de lieux touristiques littoraux

\section{Les conditions géopolitiques d'une gestion durable de l'eau : le cas du bassin rennais}

The geopolitical conditions of a durable management of the water: the case of the Rennes basin

\section{Éric Grujard}

\section{(2) OpenEdition}

\section{Journals}

Édition électronique

URL : https://journals.openedition.org/norois/92

DOI : 10.4000/norois.92

ISBN : 978-2-7535-1554-3

ISSN : $1760-8546$

Éditeur

Presses universitaires de Rennes

\section{Édition imprimée}

Date de publication : 1 mars 2008

Pagination : 91-110

ISBN : 978-2-7535-0690-9

ISSN : 0029-182X

\section{Référence électronique}

Éric Grujard, "Les conditions géopolitiques d'une gestion durable de l'eau : le cas du bassin rennais », Norois [En ligne], 206 | 2008/1, mis en ligne le 01 mars 2010, consulté le 13 janvier 2022. URL : http:// journals.openedition.org/norois/92; DOl : https://doi.org/10.4000/norois.92

(c) Tous droits réservés 


\title{
LES CONDITIONS GÉOPOLITIQUES D'UNE GESTION DURABLE DE L'EAU :
}

\section{LE CAS DU BASSIN RENNAIS}

\author{
ÉRIC GRUjARD \\ INSTITUT FRANÇAIS DE GÉOPOLITIQUE \\ (Université Paris 8), \\ 2, rue de la Liberté - 93526 SAINT-DENIS cedex \\ eric.grujard@wanadoo.fr
}

\begin{abstract}
RÉSUMÉ
En s'appuyant sur le cas du bassin rennais, cette étude de géopolitique montre que la question de la préservation de la ressource en eau ne peut plus être comprise comme une question uniquement technique mais se révèle comme le cadre où se manifestent des rivalités entre acteurs pour le contrôle de la ressource et, à travers la ressource, celui de l'usage du territoire. L'essor des procédures de concertation et de participation du public a créé de nouvelles configurations d'acteurs qui modifient les rapports de force sur les territoires et les modalités de la gestion de l'eau en France.
\end{abstract}

Mots CLÉS : Aménagement du territoire - Développement durable - Eau/politique publique - Géopolitique - Gestion des ressources en eau.

\section{ABSTRACT \\ The geopolitical conditions of a durable management of the water: the case of the Rennes basin}

This geopolitical analyis is based on the case study of the Rennes basin (Western France) and shows that the issue of the preservation of water resources can no longer be perceived as a purely technical question. It is shown to be the framework within which various political and economical bodies interfere to gain the control of the resource and, via the resource, to gain a control on local planning. The emergence of procedures for public consultation and participation have lead to new relations between these bodies. Therefore territorial power struggles and methods of water management in France are changing.

KEYWORDS : Land settlement - Geopolitics - Sustainable development - Water management - Water/Public policy.

Il n'est pas habituel d'employer le terme géopolitique pour évoquer les modalités de la gestion de l'eau en France même lorsqu'il s'agit de traiter de l'exploitation des ressources hydrauliques sous l'aspect de rivalités politiques. Pourtant, avec la très médiatisée notion de développement durable et le besoin prégnant de décrypter ce leitmotiv apparent de la décision politique, un champ s'est ouvert pour la recherche. Ainsi, l'étude géopolitique des interdépendances entre acteurs qui font les politiques publiques (Gauchon et Tellenne, 2005) peut apporter un éclairage sur l'imbroglio 
territorial au sein duquel le bassin-versant joue un rôle notoire. Depuis plus de trente ans, dans la Région Bretagne, la question de la qualité de l'eau qui mobilise fortement l'opinion publique, est devenue l'épicentre d'une géopolitique pour les acteurs de la gestion de l'eau et de l'aménagement sur plusieurs échelles de territoires (Loyer, 2005). Dans un contexte de rivalités entre acteurs de l'eau, depuis le début des années 1990, l'État, la Région, les départements, l'Agence de l'eau Loire-Bretagne unissent ostensiblement leurs efforts pour porter un programme régional de restauration de la qualité des eaux grâce à l'aide considérable de financements publics. Sous une appellation en forme de slogan, «Bretagne Eau Pure » est supposé développer une politique de l'eau ambitieuse et efficace afin d'éradiquer les pollutions endémiques qui contaminent les eaux bretonnes ${ }^{1}$. Aujourd'hui, les résultats atteints n'apparaissent pas à la hauteur de la mobilisation des moyens financiers et techniques mis en place ${ }^{2}$, et le temps du possible miracle annoncé par certains élus semble désormais révolu. Les discours explicatifs divergent selon les acteurs quant à la fragilité des résultats obtenus qui seraient, au mieux ceux d'une stabilisation de la dégradation de la qualité des eaux ${ }^{3}$ (Nicolazo et Redaud, 2007). Chacun selon les intérêts qu'il défend développe une analyse spécifique de la situation, et d'aucuns y perçoivent même un encouragement à poursuivre et accroître l'effort, « la Bretagne est manifestement décidée à trouver les meilleurs compromis permettant un développement durable grâce à un environnement de qualité ", assure la conclusion d'un rapport du Conseil économique et social de Bretagne ${ }^{4}$. Dans un contexte de décentralisation et de développement de l'intercommunalité, Rennes se caractérise par une position de métropole confrontée à des enjeux de coopérations territoriales pour la gestion de son eau. Le cycle de l'eau ne connaît pas les limites politico administratives et cette condition participe de cette problématique de planification urbaine : répondre aux besoins en eau de la population d'une agglomération en devenir et tenir compte hors métropole des intérêts d'autres populations et de leurs activités, le tout dans un contexte de grande fragilisation qualitative et quantitative des ressources en eau potable disponibles ${ }^{5}$. Cette approche qui se veut géopolitique par la démarche, s'efforce de révéler ainsi les conditions géopolitiques de la gestion de l'eau dans un espace où l'intervention de politiques publiques interroge la définition de territoires pertinents pour l'action.

\section{Le bassin rennais une entité politique primordiale pour la production d'eau potable en Ille-et-Vilaine}

\section{LES COMPOSANTES GEOGRAPHIQUES ET POLITIQUES D'UN TERRITOIRE DE L'EAU}

Le bassin rennais n'existe pas en tant que bassin hydrographique, il s'agit d'une construction politique qui correspond davantage à un bassin de vie et se confond géographiquement à la fois avec Rennes Métropole (communauté d'agglomération) et le Pays de Rennes (regroupement d'EPCI, dont Rennes Métropole) (Chardronnet, 1980 ; Le Rhun, 1988, 1994). Le bassin rennais, c'est aussi la dénomination d'usage du Syndicat mixte de production du bassin rennais (SMPBR), l'un des six syndicats mixtes de production d'eau potable du département de l'Ille-et-Vilaine. La ville de Rennes, acteur principal de ce syndicat, est propriétaire de l'ensemble des installations de production et dispose de quatre ressources : Rennes I, Rennes II, Rennes III et Rennes IV pour l'adduction d'eau potable qui desservent $54 \%$ de la population de l'Ille-et-Vilaine (450000 habitants).

1. DIREN, 1999, Atlas de l'environnement en Bretagne. La Bretagne, des hommes, un territoire, Rennes, DIREN-SEMA de Bretagne, 99 p., (document n. p.).

2. Cour des Comptes, février 2002, La préservation de la ressource en eau face aux pollutions d'origine agricole : le cas de la Bretagne, rapport au Président de la République, 44 p., (document n. p.).

3. Agence de l'eau Loire-Bretagne, 2007, La qualité des rivières dans votre département entre 2003 et 2005 : l'Ille-et-Vilaine, Rennes, DIREN-SEMA, 125 p., (document n. p.).

4. Conseil économique et social de Bretagne, juin 2003, Le défi de la qualité des eaux en Bretagne, Rennes, 467 p., (document n. p.).

5. Conseil scientifique de l'environnement de Bretagne, décembre 2005, Évolution de la qualité des eaux en nitrate dans les bassins versants Bretagne Eau Pure, Rennes, Conseil Régional, 16 p. (document n. p.). 
Le volume produit atteint 19 millions de mètres cubes par an dont plus de 6 millions de mètres cubes sont revendus à des collectivités extérieures soit sur les canalisations d'eau brute soit sur les réseaux de distribution. Toutes ces collectivités (syndicats, communes) ne sont pas membres du SMPBR. La dimension départementale de la ville de Rennes dans les infrastructures de production d'eau potable est donc incontestable.

\section{LA MENACE D’UNE PÉNURIE IMPOSE UNE NOUVELLE ORGANISATION DE L’AEP}

Le sous-sol schisteux du bassin de Rennes contraint à un approvisionnement de la ressource en eau pour $70 \%$ environ d'origine superficielle. Un rapport du Conseil général, en date du 26 avril 1996, indique que $31 \%$ des ressources d'Ille-et-Vilaine ne devraient pas être exploitées pour cause de concentration trop forte en nitrate, et $94 \%$ des ressources brutes dépassent au moins quelques jours par an les normes de potabilisation en matière de concentration en matières organiques. La moyenne annuelle des précipitations est de $600 \mathrm{~mm} / \mathrm{an}$ dans le bassin rennais, l'une des plus basses du département, et l'évapotranspiration y est forte. Le fleuve Vilaine qui traverse ce territoire, peut connaître de sévères étiages l'été et ses eaux ne satisfont pas aux normes de qualité nécessaires à leur potabilisation. L'alimentation en eau potable de la ville de Rennes s'est par conséquent organisée très tôt dans une logique hors bassin afin de pallier tant les menaces sanitaires que les nombreux conflits d'usages qui ont marqué le quotidien de la vie des Rennais jusqu'au XIX $X^{e}$ siècle.

Le Conseil général d’Ille-et-Vilaine jouera peu à peu un rôle conséquent dans la coordination de la politique d'aménagement pour l'AEP qui commencera notamment au début des années 1970 par la mise en place d'un programme d'aménagement pour la Vilaine amont dans le canton de Vitré (Allain et al., 2005). En 1988, de graves inondations touchent le département, suivies ensuite par une série de sécheresses estivales entre 1989 et 1992. En 1989, la ville de Rennes ne dispose plus que de 15 jours de réserve dans l'un de ses barrages-réservoirs. L'idée d'une organisation départementale de l'eau et d'une mutualisation des moyens commence alors à naître à cette époque entre les différents syndicats et les collectivités locales. Le contexte politique local peut donner l'apparence d'être dénué de tensions et finalement peu conflictuel, cependant le respect notoire des équilibres entre les différentes forces politiques ne doit pas donner l'illusion que des rivalités de pouvoirs n'exerceraient pas quelque influence sur les modalités de concertation et les prises de décisions en matière de gestion de l'eau (Giblin, 2003). Par ailleurs, la situation très emblématique de la question de la dégradation de la qualité de l'eau en Bretagne demeure susceptible de déterminer la moindre opération de gestion de la ressource en eau sur le territoire régional (Ménesguen, 2003). Le projet d'aménagement de nouvelles ressources en eau dans le bassin rennais n'échappe assurément pas à cette problématique. Les quatre sites de production d'eau brute du bassin rennais, gérés par le SMPBR, ne sont pas éparonés par la dégradation de qualité. Les adductions de Rennes I est réputée produire des eaux nitratées, ce qui peut imposer la fermeture de drains. Rennes II qui est une prise d'eau sur le Couesnon est nitratée comme les autres ressources superficielles et peu présenter une contamination par les pesticides. Les retenues de Rennes III et IV sont, elles, davantage concernées par les pollutions par des pesticides. La présence excessive de matière organique doit aussi faire l'objet d'une surveillance spécifique. Le mélange d'eaux de différentes origines est rendu nécessaire pour respecter la qualité réglementaire par dilution croisée des pollutions.

\section{Aller chercher l'eau sur d'autres territoires, un acte fondateur dont la légitimité suscite encore des critiques}

\section{« LES MÉTROPOLES : DES ORGANISMES QUI DÉVORENT LES TERRITOIRES!»}

Cette représentation de Rennes comme « organisme urbain » qui par « appétit territorial » dévorerait inéluctablement son environnement, est très répandue. La ville de Rennes, dès la fin du 
XIX ${ }^{e}$ siècle, confrontée à un manque d'eau crucial tant pour des raisons sanitaires que de développement économique, a déployé une logique d'approvisionnement en eau potable sur d'autres territoires, avec l'idée déjà très porteuse selon laquelle aller chercher l'eau à sa source, c'était conquérir «l'eau pure». En 1880, ce premier site de production d'eau souterraine par drains (Rennes I) couvre $32 \mathrm{~km}^{2}$ à $40 \mathrm{~km}$ de l'agglomération rennaise sur les sous bassins de la Loisance et de la Minette. L'entreprise se heurta déjà à des conflits d'usages et de territoires importants. Le 28 juillet 1880, un traité sera signé entre Le Bastard, sénateur maire de la ville de Rennes et l'ingénieur en chef des ponts et chaussées et directeur général de la Compagnie générale des eaux, par lequel la ville de Rennes confèrera à la CGE la régie intéressée de ses eaux de «toute provenance qui seront disponibles, et dont le cube quotidien pourra atteindre trois mille mètres cubes, pendant la période de cinquante années après la complète organisation de la distribution des eaux des sources de la Minette et de la Loisance ».

\section{LES TERRITOIRES DE L’EAU SONT DES TERRITOIRES ÉLECTIFS}

Ce conflit ville/campagne permet aussi d'entretenir parfois un discours plus politique au sein même du département d'Ille-et-Vilaine selon un clivage gauche/droite plus classique. Depuis l'élection municipale de 1977, le bassin rennais correspond au terrain électoral de la gauche (agglomération rennaise et cantons situés sur la métropole) alors que le Conseil général (présidé par le député maire de Vitré, Pierre Méhaignerie, jusqu'en 2001) est lui à droite (jusqu'en avril 2004). Ces deux entités politiques territoriales se distinguent singulièrement par l'émission d'un discours sur l'eau et sa gestion qui s'efforce d'être en phase avec les préoccupations de leurs électorats respectifs : urbain ou rural. La Ville de Rennes met désormais l'accent de façon très médiatique sur la reconquête de la qualité de l'eau par sous bassin-versant en développant des actions en direction des agriculteurs. Le Conseil général, dans un souci de ne pas froisser le monde agricole déjà malmené dans son ensemble par un contexte économique difficile, préfère davantage mettre l'accent sur le développement de nouvelles connexions entre les réseaux et les mélanges entre les eaux potabilisables issues des différents sites de production. La question de la gestion de l'eau sur le territoire du département de l'Ille-et-Vilaine demeure donc remarquablement sous-tendue par des implicites politiques entre ces deux ensembles potentiellement rivaux (Grujard, 2006).

\section{Créer de nouvelles ressources de production d'eau potable : une condition vitale pour une métropole en extension}

\section{L'ENJEU D'UN POUVOIR DE L'EAU...}

Dans les années 1990, l'évolution de la demande suggère un besoin supplémentaire de 5 à 7 millions de mètres cubes pour les dix prochaines années. Ces besoins sont liés à la concentration de la population dans le bassin rennais dont le pouvoir d'attraction demeure fort et constant (Ollivro, 2000, 2005a, 2005b), à l'augmentation prévisionnelle de la consommation par habitant et d'autre part aux éventualités de sécheresse et aux obligations de vidange du barrage de la Chèze (qui produit $45 \%$ de l'eau des infrastructures rennaises).

Il est remarquable que la Ville de Rennes n'ait pas transféré la compétence eau à Rennes Métropole. Ce choix indique le rapport singulier et historique des élus rennais pour ce qui apparaît comme une prérogative. La ville de Rennes demeure donc propriétaire de l'ensemble des installations de production, elle est en outre maître d'ouvrage des opérations de construction, d'extension de mises aux normes, et la Direction des services à caractère industriel et commercial assure les montages et la direction de ces opérations.

La sécheresse de l'été 1989, qui mit la Ville de Rennes en situation de risque de pénurie alors qu'elle ne disposait plus que de 15 jours d'eau de réserve dans le barrage de Saint-Thurial sur la 
Chèze, est resté cet événement de référence qui provoqua subitement la mobilisation des acteurs rennais de la gestion de l'eau.

Sécheresses et inondations sont vécus de façon habituelle comme des moments de crise qui justifient l'intervention des pouvoirs publics. Dans un premier temps, cette analyse prospective est partagée également par la Région qui préconise elle aussi dans son schéma régional AEP (1990) de développer de nouvelles ressources. Le Conseil régional de Bretagne réalise alors son Schéma Régional d'Alimentation en Eau Potable (SRAEP), qui prévoit un programme d'aménagements hydrauliques pour l'Ille-et-Vilaine. C'est dans ce double contexte de planification et de crise que prennent forme les projets étudiés.

\section{...SE CONFRONTE À DES ENJEUX DE REPRÉSENTATIONS ET DE TERRITOIRES}

Parmi les sites retenus : Gaël sur le Meu et Lassy sur le Canut (pour les sites choisis par le Bassin Rennais), mais aussi le site mythique de la forêt de Brocéliande sur l'Aff (préconisé uniquement par la Région). Si ce besoin en nouvelles ressources est partagé à différentes échelles décisionnelles (Région, Conseil général, Bassin Rennais), les élus rennais restent particulièrement engagés dans la recherche de solutions eu égard à la fonction territoriale exceptionnelle qui caractérise leur ville. Cet engagement les expose aussi particulièrement à la contestation de ces projets d'aménagements hydrauliques tant du monde agricole qui n'apprécie ni d'être dépossédé de ses terres ni soumis à des normes agri-environnementales, que des associations de protection de la nature qui entendent défendre de l'ennoiement d'un secteur de la forêt de Brocéliande (ce haut lieu de la mythologie romantique bretonne).

À la même époque, le renforcement des normes de qualité de l'eau par la Commission européenne et la réalisation de plusieurs programmes sur l'ensemble de la Bretagne pour reconquérir la qualité des eaux et maîtriser les pollutions d'origines agricoles ${ }^{6}$ interfèrent avec ce contexte de tentative de concertation entre les différentes entités politiques mobilisées dans la mise en œuvre de nouvelles actions de sécurisation de la ressource.

Le Schéma régional d'alimentation en eau potable de 1990 préconise donc la construction de nouveaux barrages et qui outre celui sur l'Aff pour alimenter le nord du syndicat Ouest 35 et Gaël sur le Meu pour pallier les problèmes de remplissage du barrage de Saint-Thurial, propose un barrage sur le Nançon, affluent du Couesnon pour pallier les problèmes d'étiage de Rennes II, et celui de Bois-Joli pour alimenter le Syndicat mixte de production de la Côte d'Emeraude au nord de l'Ille-et-Vilaine. Ce SRAEP provoque une réflexion des acteurs du Département et laisse à penser qu'un rapprochement des différents gestionnaires de l'eau serait possible (Allain et al., 2005).

\section{Une gestion territorialisée de l'eau}

\section{UNE LOGIQUE DU CHACUN CHEZ SOI...}

La Ville de Rennes est donc le producteur d'eau prépondérant avec l'influence territoriale que l'on sait lorsque en 1973, le premier syndicat mixte de production d'eau, le SYMEVAL (syndicat mixte des eaux de la Valière), est créé dans ce département. Il sera présidé par Joseph Prodhomme, conseiller général et maire adjoint (en charge de l'eau notamment) à Vitré, un homme de Méhaignerie. Le Conseil général met alors en place un vaste programme d'aménagement pour la Vilaine amont dans le canton de Vitré, avec la création de trois barrages destinés au soutien d'étiage et d'écrêtement des crues : celui de La Valière (1978), de Haute-Vilaine (1982) et de La Cantache (1995). La Cantache et la Valière ont également vocation d'alimentation en eau potable pour tout le sud-ouest du département. Déléguée depuis à la Compagnie Générale des Eaux, la gestion de

6. Il s'agit d'élaborer une vision globale des ressources en eau et d'établir des scenarii d'évolution des demandes et des ressources tandis que la Directive cadre de 1989 sur les eaux brutes et traitées renforce les normes et que se mettent en place Bretagne eau pure I (BEP I) et le plan de maîtrise des pollutions d'origine agricole (PMPOA). 
ces trois ouvrages sera confiée dans un premier temps au SYMEVAL qui coordonne désormais la gestion quantitative et qualitative des eaux sur son territoire. La logique du maître d'ouvrage est clairement formulée : il s'agit d'assurer l'autonomie de la production d'eau pour ce qui deviendra le Pays de Vitré. La représentation exprimée est celle « d'un puissant syndicat producteur d'eau potable jusqu'aux portes de Rennes... ». L'une des ambitions à peine voilée de ce projet consiste donc à concurrencer d'une certaine manière Rennes en tentant de contenir son influence voire son extension territoriale en matière de distribution de l'eau.

Un autre objectif plus technique à ce projet mais qui deviendra un principe récurrent d'une certaine façon de concevoir la politique de l'eau se justifie par l'idée qu'en « possédant la quantité, on possède aussi la qualité ». Cependant, le sursis à statuer par le Conseil Supérieur d'Hygiène de France sur la demande d'utilisation du barrage de la Cantache pour l'alimentation en eau potable en raison d'une mauvaise qualité de l'eau brute (teneurs en pesticides et nitrates notamment), fut perçu incontestablement comme une véritable déconvenue pour le maître d'ouvrage. Cette préoccupation ainsi formulée sonnera aussi le glas d'une conception très fragmentée des territoires de la gestion de l'eau au détriment d'une approche nécessairement plus globale de celle-ci. Lorsque l'étude prospective des déficits en eau survient, la gestion de l'eau est donc déjà marquée par une très forte territorialisation tandis que certaines tentatives du Préfet de Région et de la DIREN pour amener les acteurs à penser globalement la gestion de l'eau à l'échelle du bassin-versant avaient précédemment échoué. Les territoires de l'eau sont des territoires de pouvoir (Grujard, 2003), lorsque Pierre Méhaignerie, alors président du Conseil général, se résoudra finalement à organiser une départementalisation de la gestion de l'eau, cela générera des repositionnements stratégiques des différents acteurs.

\section{...SE RENFORCE ET GÉNÈRE DES MODES DE NÉGOCIATION}

Le schéma régional d'alimentation en eau potable semble très mal accepté par les élus du Département, qui entendent rester maîtres chez eux mais il aura pour première conséquence politique de provoquer en 1992 le regroupement par bassin des 66 communes ou syndicats de distribution d'Ille-et-Vilaine au sein de six syndicats mixtes de production d'eau potable et s'accompagnera de la création du Syndicat mixte de gestion du fond départemental pour le développement de la production d'eau potable en Ille-et-Vilaine (SMGD AEP 35). Cette organisation a pour objectif de mutualiser le financement des futurs moyens de production d'eau, d'en développer les infrastructures et leur protection, même si ce dernier point semble avoir été longtemps délaissé. Le financement de ces programmes est organisé sur la base d'une surtaxe de 50 centimes de francs ( 0,076 centimes d'euros) par mètre cube consommé payables par les usagers. Cependant, autre choix politique, le SMGD n'a pas le statut juridique pour être maître d'ouvrage: chaque syndicat mixte reste donc maître chez lui. Le Syndicat mixte de production du bassin rennais (SMPBR) s'est alors constitué pour répondre à cette dynamique du SMGD qu'a initiée le Conseil général. Rapidement, les premières négociations, un peu délicates, ont concerné la question de la représentativité de chaque syndicat dans les instances du SMGD. Le SMPBR revendiquera un représentant par tranche de volume d'eau produit. L'application de ce principe permettra une composition représentative constituée de quatorze délégués dont quatre pour le collège du Conseil général, dix pour le collège Syndicats de production, dont quatre pour le bassin rennais. Le SMGD est d'emblée présidé par le président du Symeval, tandis que le SMPBR est présidé par un élu communiste de la municipalité rennaise. Les communistes, depuis plusieurs mandats, occupent le poste de conseiller municipal chargé de la gestion de l'eau, et Jean-Michel Héry a succédé à Christian Benoist après les municipales de 2000. Cependant, ces dernières ont été suffisamment favorables à la gauche pour provoquer des remaniements politiques au sein de certains syndicats mixtes et permettre l'élection de Jean Michel Héry à la présidence du SMGD. Certaines dissensions ont alors émergées avec ce changement. Deux logiques semblent désormais s'affronter. L'une qui serait prétendue « de droite », consisterait à favoriser la création de « toujours plus de tuyaux » 
en développant les interconnexions, lorsque l'autre qui serait elle prétendue « de gauche » (sic!) et viserait à protéger la ressource en eau par des programmes de reconquête de sa qualité. Sur les 50 centimes de francs ( 0,076 centime d'euros) alors consacrés aux infrastructures, $20 \%$ doivent désormais être utilisés pour cette reconquête de qualité (par exemple financer les Contrats territoriaux d'exploitation (CTE), ou encore les périmètres de protection de captage, source de grandes tensions dans le monde rural).

Il faut mettre ces deux logiques, qui se veulent opposées, en perspective avec les programmes développés par la Ville de Rennes sur les bassins versants de ses quatre zones de production d'eau. Lorsque celles-ci se situent dans les aires d'influence politique de la droite du Conseil général, cette intrusion territoriale n'est pas toujours bien vécue par certains élus. Force est de constater que cette conjoncture crée une situation de proximité qui est de matière à favoriser les actions de concertation entre l'ensemble des acteurs sur les bassins versants concernés. Une véritable convergence d'intérêt semble s'être dégagée qui fait suite pourtant à un long contentieux entre le monde rural (agriculteurs) et la Ville de Rennes. Avec l'aide de la SAFER, comme opérateur foncier, mais aussi du maire de la commune concernée et du responsable professionnel agricole local, la Ville de Rennes a ainsi acquis la maîtrise de 140 hectares dans le périmètre de protection rapprochée de son captage de Rennes I dont 90 ha ont été boisés.

\section{Vers un changement de logique territoriale?}

\section{LA CONTROVERSE D'UNE DÉLICATE MAîTRISE D’OUVRAGE...}

L'épisode décevant de la prise d'eau du barrage de la Cantache (mis en eau en 1995), serait un élément déclencheur du renoncement du Conseil général (Méhaignerie) à se porter maître d'ouvrage de nouveaux barrages. Pourtant le Bassin rennais réclame concomitamment l'engagement et donc la participation financière du Conseil général en particulier pour le projet de Gaël sur le Meu dont une partie du bassin-versant est située en Côtes-d'Armor. Ce projet qui apparaît comme un enjeu politique important, sera un objet de négociation entre le Bassin rennais et le Conseil général. Il suscite l'opposition de la profession agricole pour des raisons de déperdition foncière mais aussi eu égard à la mauvaise qualité des eaux du Meu, qui ferait craindre de nouvelles contraintes réglementaires sur les pratiques agricoles sur l'ensemble du bassin-versant (Martin, 2003). Le Conseil général rechigne à s'engager dans ce projet dont Rennes serait le principal bénéficiaire. Le SMPBR (dont Rennes est l'acteur dominant), estime lui que le Conseil général doit fortement s'impliquer dans ce projet à la fois compte tenu de sa dimension interdépartementale mais aussi parce qu'il est plus délicat de le défendre aux yeux de ses détracteurs en étant identifié exclusivement comme « rennais ». Le SMPBR provoque ainsi un embarras certain du Conseil général en lui renvoyant le débat sur la prospective des déficits en eau et la création de nouvelles ressources pour l'AEP.

\section{...STIMUle LA RECHERCHE DE SOlutions ALTERNATIVES NÉGOCIÉES}

En effet, dans un contexte propice à la négociation, l'émergence d'une solution alternative à la construction d'un barrage fait son chemin avec la possibilité d'une interconnexion par une prise d'eau alimentant le barrage de Saint-Thurial. Le débat qui s'est en effet engagé entre le Conseil général et le Conseil régional sur les risques réels de déficits en eau, conduit entre 1994 et 1996 à faire réactualiser le SRAEP 1990 par un expert local reconnu ${ }^{7}$ (le cabinet Saunier Techna).

L'étude confirme les conclusions du SRAEP 90 sur le manque de sécurité du bassin rennais mais avec une estimation revue à la baisse (de l'ordre de 1,5 million de mètres cubes). Elle confirme

\footnotetext{
7. Cette étude réalisée par un cabinet qui travaille étroitement avec les syndicats de production d'eau du département qui ne possèdent pas d'expertise technique propre, vise à confirmer ou infirmer les estimations des déficits jugés peu crédibles demandera 20 mois supplémentaires.
} 
également les risques importants de dégradation de la ressource par les nitrates et la matière organique, et rappelle la solution d'un barrage sur le Meu à Gaël notamment en cas de sécheresse prolongée ou de vidange du barrage de Saint-Thurial. Toutefois, elle propose une solution alternative à la construction d'un barrage avec la proposition d'une prise d'eau alimentant le bassin du Chèze et donc indirectement Saint-Thurial.

Le Conseil général conçoit la possibilité de ces deux solutions mais en indiquant sa préférence pour l'interconnexion. En avril 1996, les délibérations du Conseil général aboutissent à une série d'accords pour la réalisation d'un barrage sur le Meu mais dont la maîtrise d'ouvrage serait assurée soit par le Conseil général soit par le syndicat Ouest 35 car le site se trouve dans le bassin de ce syndicat. Un avis défavorable est par contre émis pour les barrages sur l'Aff et sur le Canut (à Lassy) pour des raisons d'incompatibilité avec la politique environnementale du Département.

En juin 1996, pris entre la nécessité de transposer le SRAEP à l'échelon départemental, la demande pressante des élus rennais pour prendre une décision (le SMPBR renouvelle sa demande d'une maîtrise d'ouvrage départementale pour le barrage de Gaël), et les interpellations des opposants aux projets de barrages (monde agricole, associations écologistes), le Conseil général ne peut se résoudre à prendre seul une telle décision dont le contrôle lui échappe. Il décide d'instaurer une commission départementale pluripartite pour réexaminer l'ensemble des analyses et des solutions envisagées. Celle-ci se compose des protagonistes habituels : Conseil général et SMPBR, DDAF, DIREN et Agence de l'eau Loire-Bretagne, mais aussi de l'influente association Eaux et Rivières de Bretagne.

Cette commission rend son rapport presque un an plus tard en mai $1997^{8}$. Du fait de l'hostilité locale au projet de barrage, la commission privilégie de nouvelles solutions techniques : une prise d'eau et des interconnexions entre bassins et notamment la possibilité pour le Symeval, dès lors que la mise en exploitation pour la production d'eau potable du barrage de la Cantache sera autorisée, de fournir 2 à 5 millions de mètres cubes d'eau vers le SMPBR via une interconnexion. Elle fait aussi valoir que les risques de déficit du bassin rennais sont en partie dus aux ventes d'eau qu'il effectue à d'autres bassins.

En juin 1997, le SMPBR conteste ces conclusions en arguant qu'il est obligé de respecter ses contrats de livraison d'eau vers d'autres communes et que ce rapport ne tient pas assez compte des situations exceptionnelles de sécheresse prolongée, comme cela a été le cas de 1989 à 1990. Par ailleurs, un barrage sur le Meu pourrait remplir trois fonctions : alimentation en eau potable, écrêtage des crues et soutien d'étiage, et permettrait de limiter la multiplication des ouvrages tout en remplissant les objectifs du SDAGE.

Malgré ces critiques, le Conseil général approuve les conclusions de cette commission tout en précisant la nature des aménagements hydrauliques à envisager : la construction d'un barrage sur le Nançon pour soutenir l'étiage de Rennes II, rechercher la potabilisation des eaux de la Cantache et de Bois Joli (pourtant très dégradées), développer les interconnexions entre les grands bassins, envisager éventuellement une simple prise d'eau sur l'Aff pour alimenter le barrage de Saint-Thurial (Rennes IV). Par contre, la réalisation d'un barrage sur le Meu n'est plus retenue.

\section{L'ARBITRAGE D’UNE EXPERTISE DE CORPS DE L'ÉTAT}

Face à l'impossibilité de construire une décision consensuelle et à la pression sans relâche des élus et conseillers généraux rennais, le Conseil général décide de faire appel à l'intervention d'une mission d'expertise interministérielle pour qu'un avis soit donné quant au choix de sites des barrages de Gaël sur le Meu et sur l'Aff en forêt de Paimpont destinés à l'alimentation en eau potable de l'Ille-et-Vilaine et plus particulièrement de l'agglomération rennaise. Cette mission d’inspection conjointe, composée de deux ingénieurs du Génie rural et des Eaux et Forêts représentants respectivement le ministère de l'Agriculture et le ministère de l'Environnement, apportera

8. Schéma d'actualisation de l'alimentation en eau potable en Ille-et-Vilaine. 
cependant de nouvelles solutions qui n'avaient jamais été ouvertement proposées. Elle proposera des solutions locales pour combler le déficit et, outre l'interconnexion du Symeval avec le Bassin Rennais de faire davantage appel aux installations du barrage d'Arzal (situé dans le Morbihan dans l'estuaire de la Vilaine).

Les conclusions de cet arbitrage laisse à penser qu'une coopération naissante pourrait mettre fin aux tensions qui perdurent depuis si longtemps. Ce nouveau projet est justifié en l'habillant du thème de « la solidarité territoriale ». Trois acteurs politiques essentiels semblent y trouver leur compte : le Bassin rennais, le Conseil général et l'Institut d'aménagement de la Vilaine (IAV).

Le rapport fournit par la mission interministériel propose que l'IAV fournisse davantage d'eau en direction de l'Ille-et-Vilaine de sorte que la Ville de Rennes réduise ses fournitures d'eau. Ceci permet au Conseil général de souligner encore une fois la responsabilité du Bassin rennais dans les déficits en eau du département par sa politique de vendeur d'eau brute, en particulier lorsque ses ventes d'eau aux collectivités sont supérieures aux contrats signés avec celles-ci.

Le SMPBR demande, lui, l'approfondissement des propositions telles qu'une prise d'eau sur l'Aff, et un renforcement à partir du barrage d'Arzal, mais ne renonce pas au projet sur le Meu qu'il souhaite voir intégré dans le cadre du SAGE Vilaine afin d'écrêter les crues de la Vilaine, mais également comme solution ultime pour l'AEP.

\section{UN ACTEUR DE L'EAU LONGTEMPS MINORÉ : L'INSTITUT D’AMÉNAGEMENT DE LA VILAINE}

L'IAV est un établissement public territorial de bassin (EPTB), créé en 1961 afin de développer de grands projets d'aménagements sur la Vilaine aval et de lutter contre les risques des inondations sous l'action cumulée des marées et des crues de la Vilaine. L'IAV dont le siège se situe à La Roche-Bernard, regroupe des élus des conseils généraux du Morbihan, de l'Ille-et-Vilaine et de la Loire Atlantique. Cependant, une mésentente avec les élus de l'Ille-et-Vilaine ne permit guère le développement de l'influence de cet établissement au-delà de Redon et finalement l'IAV concentra son action principalement sur l'alimentation en eau du littoral atlantique du Morbihan et de la Loire-Atlantique soumis à une forte demande estivale, en se constituant comme maître d'ouvrage puis gestionnaire du barrage d'Arzal (construit en 1970) et de l'usine de potabilisation de Férel. Une convention signée avec les communes du littoral garantit l'exclusivité de l'approvisionnement en eau durant l'été. Le projet d'interconnexion entre Férel et le bassin rennais apparaît comme une opportunité formidable afin d'impulser les activités de l'IAV et le développement de son influence en direction de l'ensemble du bassin de la Vilaine.

Afin de conquérir la reconnaissance d'une légitimité politique sur l'ensemble du bassin de la Vilaine, l'IAV sera choisi comme structure porteuse du SAGE Vilaine. La réalisation de ce SAGE qui est par sa taille $\left(11000 \mathrm{~km}^{2}\right)$ le plus grand de Bretagne mais aussi l'un des plus grands de France apparaît comme une véritable gageure. La Commission locale de l'eau (CLE) sera présidée par Pierre Méhaignerie.

Pierre Méhaignerie a cédé la présidence du Conseil général, en 2001, à Marie-Joseph Bissonnier. Celui-ci est l'élu d'un canton dont le territoire se situe au cœur de certains enjeux puisqu'il englobe précisément une partie du bassin de l'Aff, le site de la forêt de Brocéliande et Saint-Thurial (Rennes IV). C'est Marie-Joseph Bissonnier, alors l'un des administrateurs de l'IAV, qui en alternative à l'abandon d'une prise d'eau sur l'Aff (préconisée par la mission ministérielle plutôt qu'une retenue), proposera officiellement l'interconnexion du bassin rennais avec l'usine de Férel près du barrage d'Arzal, même s'il est vrai que la conception de ce projet potentiel aura été préalablement pensée au sein des services compétents du Conseil général.

\section{LES FINALITÉS POLITIQUES DU SAGE VILAINE}

L'idée d'un SAGE sur la Vilaine est née en application de la loi sur l'eau de 1992. C'est la DIREN de Bretagne de l'époque qui en fera la demande auprès du Préfet de Région. Il s'agit de 
relancer une précédente tentative de l'État pour un projet de gestion globale à l'échelle du bassin. Le déroulement des faits incite à penser que l'État semble le seul acteur en mesure d'impulser un traitement des problèmes de gestion de l'eau sur un territoire aussi vaste et partagé entre plusieurs départements et deux régions. Les arguments employés par la DIREN, pour tenter de sensibiliser les acteurs locaux à l'interdépendance des problèmes à résoudre, sont les inondations et la qualité de l'eau destinée à l'AEP.

D’emblée, le montage politique de cette opération apparaît comme une gageure. Il semble hypothétique d'envisager qu'une véritable concertation soit menée entre tous les acteurs concernés par cette procédure appliquée à un aussi vaste territoire soumis à une pression sociale aussi forte. Pierre Méhaignerie souhaite que ce SAGE ne sombre pas dans les atermoiements ordinaires. La notion de bassin-versant comme territoire naturel de la gestion de l'eau peut en effet paraître quelque peu illusoire au regard des nombreuses et complexes interrelations qui existent déjà avec d'autres bassins versants. Cette montée en puissance soudaine et opportune de l'IAV suscite donc quelques interrogations quant aux stratégies menées par les différents protagonistes et en particulier ce qui a permis leur rapprochement.

Pierre Méhaignerie souhaite agir rapidement car trop de sujets sensibles sont susceptibles d'être évoqués ou ravivés. En effet, dégradation de la qualité des eaux de surface vis-à-vis de l'enjeu de l'alimentation en eau potable, risques d'inondations par manque d'application des Plans de prévention des risques (PPRI), conflits d'usage dans l'estuaire de la Vilaine suite aux transformations du milieu liées à la création du barrage d'Arzal : les problèmes à gérer ne manquent pas. La notoriété politique de Pierre Méhaignerie est envisagée comme un élément facilitateur de la réalisation de ce SAGE.

Cependant, rompant d'une certaine façon avec son ambition de proximité avec les problématiques locales et de consultation participative, cette CLE composée de 60 membres fonctionnera à deux niveaux. Une commission permanente de 16 membres issus des trois collèges habituels : élus, usagers et État. La constitution de cette commission permanente ayant délégation de pouvoir est supposée permettre un fonctionnement plus souple. Mais il faut bien reconnaître un certain encadrement des instances de concertation de ce SAGE où finalement toutes les décisions seront prises entre acteurs politiques, institutionnels et organisationnels déjà, eux, en grande proximité.

Le deuxième niveau sera constitué de trois commissions locales, dites géographiques : Vilaine amont, Vilaine aval et Oust. Les commissions géographiques sont supposées contenir les sources supposées de retards ou de contretemps dues aux aléas du contexte de pression sociale qui accompagne cette démarche. On assiste donc à la mise en place d'une politique de « proximité » à géométrie variable selon la nature des enjeux, ce qui au passage apparaît comme une déclinaison singulière du principe de gestion intégrée.

Si la concertation telle qu'elle a été menée lors de l'élaboration de ce SAGE semble avoir remporté le crédit des associations, parmi celles-ci, certaines s’inquiètent néanmoins que la CLE n'ait pas été saisie des travaux déjà réalisés. Par ailleurs, la question de la qualité des eaux superficielles n'a étonnamment pas véritablement fait l'objet d'études et de débats approfondis. La CLE a simplement entériné les programmes existants, justifiant qu'il fallait laisser les acteurs locaux se mobiliser par sous bassin-versant. On peut y voir une absence de volonté politique d'aborder un sujet aussi sensible qui traduit l'embarras des élus locaux dans cette région où l'agriculture intensive est la source majeure de pollutions. Ce SAGE semble pourtant confirmer une nouvelle logique : «garder l'eau sur son bassin-versant » et, de surcroît, mettre Rennes en interconnexion avec Arzal.

\section{« LA GRANDE BOUCLE DE LA SOLIDARITÉ DÉPARTEMENTALE »}

Ce projet d'interconnexion qui suppose un apport régulier et important pour assurer le «bon fonctionnement des tuyaux» et donc à des achats contractuels d'eau importants, suscite chez les techniciens rennais une crainte de la baisse de production du SMPBR qui pourrait se voir ainsi 
marginalisé voire dépossédé de son rôle de producteur d'eau prépondérant. Il faut noter aussi que la question du partage de la maîtrise d'ouvrage des interconnexions a fait l'objet de fermes négociations entre les syndicats concernés, chaque syndicat restera finalement maître d'ouvrage des installations passant sur son territoire. Pourtant, le changement de logique territoriale de ce schéma départemental d'alimentation en eau potable ne révèle pas d'évolutions remarquables quant aux représentations des enjeux et des intérêts politiques des acteurs. Cependant, le président du Syméval, Joseph Prodhomme, manifesta des velléités de remise en question des accords d'interconnexions réciproques entre son syndicat et le SMPBR. Il semblerait que l'autorisation à potabiliser les eaux de la Cantache tarde à se concrétiser et que la reconquête de la qualité des eaux brutes de ce sous-bassin versant ne soit finalement pas à la hauteur des espérances et des attentes au point qu'elle fasse désormais figure « d'Arlésienne ». Cette situation contrarie de plus en plus cette argumentation qui permettait d'envisager « la vente d'eau » du Syméval en direction du SMPBR et conséquemment de modifier les rapports de forces entre ces deux territoires de l'eau.

Les rivalités d'influences pourraient bien avoir la peau dure si l'on en juge par ces tensions qui, en décembre 2004, subsistent toujours entre ces deux entités politiques. Ce réseau d'interconnexions est appelé : " la grande boucle de solidarité départementale », mais difficile d'apprendre auprès des techniciens dans quel sens doit s'écouler cette solidarité, pour eux : "C'est l'affaire des élus! »Des raisons sanitaires ${ }^{9}$ seront finalement invoquées pour justifier que les transferts d'eau ne puissent se faire que du Syméval vers le SMPBR. Joseph Prodhomme pourra donc «vendre de l'eau aux Rennais », une expression que Jean-Michel Héry corrigera par « reversement d'une indemnité de coûts de production », une représentation, décidément, quelque peu divergente des enjeux entre ces deux acteurs de la gestion de l'eau (fig. la et 1b).

Effectivement, début 2006, de nouvelles dissensions continuent d'empêcher la réalisation de ce projet dont le Département de l'Ille-et-Vilaine semble vouloir se désengager, il est vrai que depuis que la gauche a remporté les élections cantonales de 2004, l'équilibre des rapports de forces qui imposait cette recherche de consensus entre l'ensemble des acteurs, s'est rompu révélant ainsi d'autres rivalités (Giblin, 2005).

Le SMPBR s'oriente désormais vers d'autres possibilités de développement de ses capacités de production d'eau potable que lui permettrait à la fois une révision à la baisse de ses besoins qu'il reconnaît avoir sans doute surestimés, et une mobilisation plus performante de ses moyens de production existants qui lui permettrait de récupérer globalement ainsi 5 millions de mètres cubes tandis que l'achat contractuel de 6 millions de mètres cubes d'eau sont nécessaires pour des raisons sanitaires déjà évoquées. Le SMPBR est accusé de « jouer le jeu de la CGE » et de négliger l'intérêt général du bassin-versant, tandis que du côté du bassin rennais on estime qu'il n'est pas répréhensible de s'entendre avec «son partenaire ». La politique de bassin-versant est pourtant ce grand credo porté par le nouveau Conseil régional de Bretagne, qui lui aussi est désormais dirigé par une majorité de gauche et cherche à impulser la généralisation des SAGE, un dispositif présenté étonnement comme novateur, mais surtout, une condition jugée nécessaire afin de recevoir ensuite des aides à la réalisation de projets de reconquête de la qualité de l'eau sur ces territoires naturels que sont les bassins versants bretons. Pourtant, l'appartenance à une même sensibilité politique ne rapproche pas le Conseil général et le Conseil régional sur les questions de politique de l'eau. Le Conseil général de l'Ille-et-Vilaine, et en particulier ses services techniques qui depuis des décennies conduisent sur leur territoire les actions de politique de gestion de l'eau et qui semblent rétifs à se soumettre à une conception régionale de l'eau. La Région est tentée de se tourner alors vers l'IAV qui demeure un interlocuteur un peu mieux écouté, en tout cas moins illégitime aux yeux de certains acteurs, et qui pourrait jouer ce rôle médiateur qui fait décidément défaut à ce territoire. Un rôle qui sied d'ailleurs fort bien à l'IAV qui ne peut qu'être conforté par une telle sollicitation.

9. L'eau doit circuler de façon permanente et en quantité suffisante afin de garantir un bon état de qualité sanitaire et éviter le développement d'une activité microbienne. 

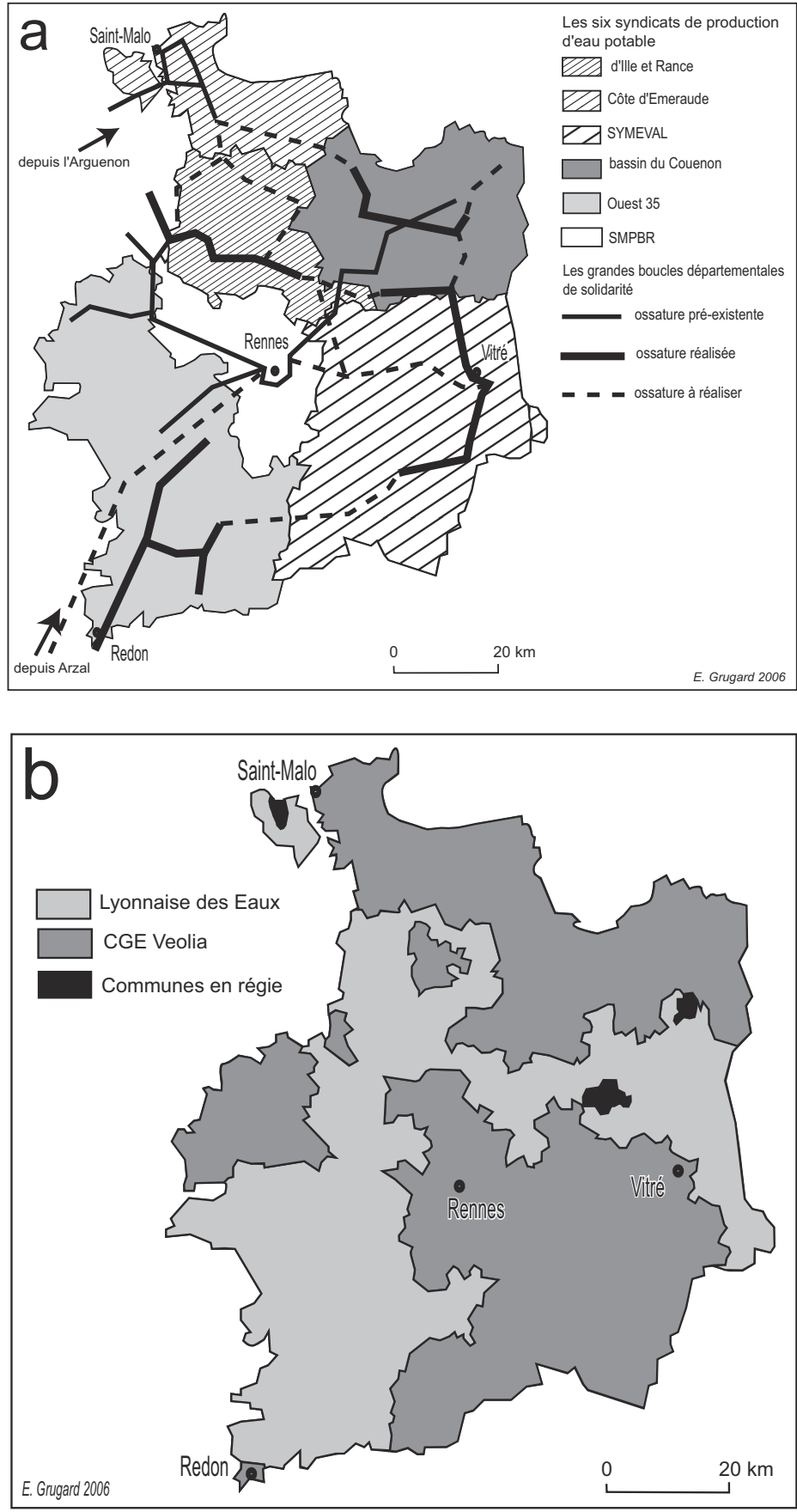

Figure 1 : Une forte territorialisation de l'eau en Ille-et-Vilaine

la : Les six syndicats de production d'eau potable et les grandes boucles départementales de solidarité; lb : Le partage territoriale des compagnies fermières.

$$
\text { A strong territorialisation of the management of water in Ille-et-Vilaine }
$$

1a: Six syndicates of production of drinking water and the big departmental loops of solidarity; 1b: The territorial division of compagnies. 


\section{L'enjeu du partenariat public/privé}

\section{LES IDÉOLOGIES À L'ÉPREUVE D’UNE GESTION DURABLE DE L'EAU}

C'est bien au nom des fondements du développement durable qu'une remise en cause de la délégation du service public de l'eau surgit et devient l'objet d'un enjeu politique. Des mouvements politiques affirment l'incompatibilité de ce mode de gestion avec le souci d'une bonne gouvernance de l'eau. Durant l'année 2004, le contrat de Véolia avec Rennes qui arrivait à échéance, a fait l'objet d'une renégociation tandis que la gestion en régie faisait simultanément débat au sein du conseil municipal. Depuis la signature du dernier contrat, les règles ont changé : la loi Sapin de 1993 oblige désormais les collectivités locales à organiser une mise en concurrence pour l'attribution du marché de l'eau et à instaurer des commissions consultatives des services publics locaux. La Ville de Rennes a donc examiné les offres de plusieurs entreprises privées. Les négociations ont ensuite été poursuivies avec Véolia qui proposait la meilleure offre technique et financière et le nouveau contrat a ensuite pris effet au premier janvier 2005. La majorité municipale de gauche a abordé de manière très divisée le dossier de la gestion de l'eau potable et, à l'issue d'un débat houleux entre les tenants de la municipalisation (Verts, Radicaux de Gauche, Union Démocratique Bretonne) et partisans du système de délégation (Socialistes, Communistes), le conseil municipal a décidé de confier la gestion du marché de l'eau à Véolia. Cette volonté d'une gestion en régie municipale a surtout fait l'objet d'un enjeu politique important pour les Verts de Rennes en vertu de l'application du principe : «Penser global, agir local... : contre les multinationales et la loi de l'argent. » Ceux-ci ont réalisé une campagne très médiatisée sur ce thème de prédilection du courant altermondialiste qu'est la privatisation de ce bien commun qu'est l'eau et de sa « marchandisation » (Pétrella, 2003). Cette campagne s'est orchestrée autour du thème de la reconquête de « l'eau pure » à un moment où se décidait simultanément l'autonomie des Verts plutôt que l'alliance à gauche pour le scrutin des élections régionales. Cependant, au-delà de ces jeux d'acteurs très stratégiques, la question reste posée de la gouvernance de l'eau et en particulier de l'incontournable influence du délégataire sur les processus décisionnels au sein des autorités publiques (Giblin, 2007).

\section{LE POIDS D’UN ARGUMENTAIRE ÉCONOMIQUE}

Si dans le cadre d'une délégation de service public la société fermière s'engage à produire et à distribuer l'eau potable en utilisant les équipements dont la Ville de Rennes reste propriétaire, elle doit veiller à l'entretien et au renouvellement des canalisations. Un service municipal, la Direction des services à caractère industriel ou commercial (SCIC), créé à Rennes dans les années 1970, est chargé du contrôle de la société, sur les plans financier, économique et sanitaire. La Ville de Rennes a affirmé d'emblée ne pas avoir d'a priori dans le choix entre régie et délégation de service public puisqu'elle utilisait déjà les deux systèmes : délégation pour l'eau et régie pour l'assainissement. Aussi, pour choisir en toute connaissance de cause, elle a fait réaliser une étude de faisabilité du système de régie afin de la confronter aux résultats des négociations avec les prestataires privés. L'enjeu économique était considérable car si depuis une dizaine d'années, le nombre d'abonnés (ce sont à $99 \%$ des particuliers) apparait stable, ceux-ci ne consomment que $57 \%$ des volumes facturés et l'autre partie l'est par les communes voisines et les industriels. Par ailleurs, $35 \%$ de l'eau produite sont vendus à des collectivités voisines, une part en nette progression depuis quelques années.

L'argument économique a fortement joué en faveur du renouvellement du contrat avec la compagnie délégataire dont la durée est passée de 16 à 10 ans et le cahier des charges est devenu plus rigoureux que le précédent. Il oblige le délégataire à justifier ses charges, ses choix d'investissements et introduit une batterie d'indicateurs chiffrés pour juger de la bonne exécution de la prestation. Le montant inscrit pour le renouvellement des différents ouvrages (canalisations entre autres) a été majoré de $37 \%$. L'exécution de ce programme (39 millions d'euros de travaux sur 10 ans) est 
très encadrée, avec notamment un retour des excédents éventuels à la Ville. La rémunération de la CGE baisse de 9,4\% ce qui aura pour conséquence (toutes choses égales par ailleurs, et sur la base d'une consommation de $120 \mathrm{~m}^{3}$ ), une baisse de 4,1\% sur la facture de l'usager.

La comparaison entre le coût du service en régie et celui de la Générale des Eaux faisait apparâ̂tre un écart de 2,6\% en faveur de la régie. La Ville a jugé cette différence trop minime. D'autant qu'il s'agissait de comparer un prix garanti (offre ferme de la CGE) avec un coût prévisionnel qui serait sans doute dépassé dans le cas d'une régie à créer de toutes pièces. Elle a par ailleurs estimé que ce surcoût était largement compensé par la plus-value offerte par une grosse société disposant de moyens importants en matière de recherche-développement ou de service aux clients, activités qui n'ont pu être chiffrées dans la perspective d'une gestion en régie.

Quand on compare le prix de l'eau en France, on constate qu'il n'y a pas de corrélation avec le mode de gestion choisi. Ce prix dépend bien davantage de la qualité des eaux brutes, des facilités de prise d'eau, de stockage et bien sûr de la distance entre la source et les usagers. De ce point de vue, Rennes n'est pas très avantagée mais obtient cependant un prix au $\mathrm{m}^{3}$ (2,60 euros) se situant dans la moyenne nationale.

\section{LES STRATÉGIES DES GRANDES COMPAGNIES D’EAU}

Les nouvelles normes européennes, l'obligation de traitement des eaux usées par les communes de 2000 habitants ou bien encore la mise en œuvre d'un programme de maittrise des pollutions d'origine agricole, ont des conséquences financières dont l'ampleur laisse envisager des marges de rentabilité considérables pour les opérateurs qui s’impliquent dans le créneau du développement durable. Face au double enjeu du maintien de la sécurité sanitaire et du manque de disponibilité en eau potabilisable, le positionnement stratégique des compagnies d'eau internationales (Suez Ondéo, Veolia, Saur), s'applique à satisfaire aux fondements du développement durable par un investissement d'efforts de financement de pôles de Recherche et Développement. Le coût croissant des infrastructures et des développements technologiques présente une opportunité considérable aux investisseurs mais le sujet sensible d'une tarification adéquate des services d'eau et d'assainissement participe également d'une démarche de développement durable des principaux acteurs de ce secteur. Il s'agit de faire concilier la période longue d'amortissement qu'impose la récupération des investissements avec les aléas provoqués par l'interférence d’intérêts politiques du moment. Les contextes géopolitiques qu'ils soient locaux ou internationaux sont désormais des données incontournables pour les grandes compagnies d'eau. Lors de la renégociation des contrats, ces opérateurs privés doivent donc anticiper les tentatives locales d'une remise en question du modèle de gestion déléguée afin de minimiser leurs risques financiers. comme à Rennes, l'interférence des positionnements idéologiques supposés créer un rapport de force politique, peut avoir pour conséquence d’infléchir le rapport de force en défaveur du délégataire.

\section{Les territoires de l'eau comme territoires de pouvoirs}

La victoire de la gauche aux scrutins locaux de 2004, cantonales et régionales ne semble pas avoir généré ensuite de grandes modifications dans la façon d'aborder les questions de la gestion de l'eau. La Région Bretagne a déployé une attitude très volontariste en réclamant en vain que la nouvelle loi sur l'eau (2006) retienne sa demande d'expérimentation d'un rôle de " chef de file » de la gestion de l'eau. Ce qui permet encore d'alimenter cette polémique qui perdure entre les régions et le gouvernement sur les conditions de la décentralisation.

Parmi les «mesures phares » mises en œuvre par le nouvel exécutif régional la prise en compte du bassin-versant comme nouveau territoire de projet est présenté comme essentielle. Pourtant, au regard de l'ampleur des conflits qui se déroulent en Bretagne entre lobbies économiques et/ou agricoles, entre syndicats professionnels agricoles, entre écologie politique et partis traditionnels, pour ne citer que les plus courants, il est permis de s'interroger quant au réel poids politique du 
bassin-versant comme territoire de pouvoir. La très vertueuse territorialisation des politiques de l'eau peine à cacher combien cette tentative de construction de solidarités à l'échelle du bassinversant résiste assez mal à l'épreuve de l'affrontement des divers intérêts économiques, politiques et autres rivalités de territoires de l'eau.

Les débats qui précédèrent le lancement d'un SAGE sur la Vilaine, furent une occasion parmi d'autres afin de remettre en question la pertinence du territoire du SDAGE Loire-Bretagne et regretter qu'il n'y ait pas plutôt un SAGE Bretagne ou un SRAGE (Schéma régional d'aménagement et de gestion des eaux). L'argument récurrent consiste à préférer qu'il soit procédé à des découpages géographiques dits «plus fins » pour résoudre les problèmes qui se posent. Cette finesse revendiquée d'un territoire pertinent pour agir marque le souci de contrôle politique de l'action à engager.

Tous les partis politiques souhaitent désormais cette expérimentation de la gestion de l'eau à l'échelon de la Région Bretagne, ce thème semble très porteur et ne peut être que payant politiquement pour celui qui le portera au plus haut, quitte à faire un peu de surenchère. La Région, sous présidence de Josselin de Rohan (UMP), justifiera en son temps sa candidature à une expérimentation de la décentralisation dans le domaine de l'eau selon l'argument géographique de l'homogénéité hydrographique. D'aucuns s'interrogent quant à savoir si la Bretagne nécessiterait des normes spécifiques pour ses eaux brutes. En outre, ce Schéma régional d'aménagement et de gestion des eaux poserait la question de ses modalités financières puisque aujourd'hui la Bretagne est la principale bénéficiaire des aides financières prodiguées par l'ensemble de l'Agence de l'eau Loire-Bretagne. Le sénateur Ambroise Guéllec (UMP), président du Comité de bassin LoireBretagne, est alors vice-président de la Région Bretagne, il n’ignore pas toutes ces conditions mais soutient pourtant, lui aussi, ce projet. Profitant d'un climat politique rendu propice par le grand dessein politique du Premier ministre Jean-Pierre Raffarin en matière de décentralisation, mais aussi de sa proximité avec Roseline Bachelot, supposée faire évoluer le projet d'une grande loi sur l'eau, il s'attachera à faire avancer cette vision d'une régionalisation de l'eau.

On comprend l'importance que représente cette nécessité stratégique pour la droite de la Région Bretagne d'incarner ce fameux « chef de file » de la gestion de l'eau. Un schéma breton de l'eau placé sous la responsabilité de la Région qui organiserait le débat permettrait de contrôler les actions à mener et les financements qu'elles drainent, mais aussi la communication que cette position suppose, et tenter ainsi de maîtriser ce qui est devenu un vecteur potentiel de déstabilisation politique.

Depuis les bons résultats de la gauche à l'issue des cantonales (Ille-et-Vilaine et Loire-Atlantique) et des régionales (Bretagne et Pays de Loire) de 2004, de nouvelles rivalités d'influences au sein de certaines structures de gestion de l'eau n’ont pas manqué de se révéler.

Ainsi, la présidence de l'IAV a été prise par un socialiste du canton de Saint-Nicolas-de-Redon situé dans le département de la Loire Atlantique. Puis, Jean-René Marsac, conseiller régional socialiste et conseiller municipal à Redon s'est lui emparé de la présidence de la CLE du SAGE Vilaine au détriment d'une conseillère générale (UMP) du canton d'Allaire dans le Morbihan et mairesse de Saint-Vincent-sur-Oust. Il est remarquable que ces trois personnalités politiques ont en commun d'appartenir au territoire de la communauté de communes du Pays de Redon (fig. 2).

Le Pays de Redon, situé aux confins de trois départements et de deux régions, apparaît subitement comme une zone d'interface politique dictée par les contraintes de l'hydraulique du fleuve Vilaine. Il s'agit d'un espace circonscrit d'influence de l'eau dans de nouvelles politiques territoriales accompagnant des mesures de prévention et de protection contre les crues. Des projets de développement économique suspendus à de nouveaux aménagements hydrauliques dans cet espace ont donc révélé des enjeux politiques locaux et des rivalités conséquentes pour leur contrôle. Il faut aussi rappeler que le découpage des départements qui a eu pour résultat de situer toute une partie des affluents de la rive gauche de la Vilaine aval en Loire-Atlantique (et soit dit en passant hors de la Bretagne), nécessite un véritable effort de coordination entre trois services départementaux distincts lorsqu’il s'agit d'alerter les populations contre l'apparition de crues du fleuve, une condition qui dans le passé n'a pas semblé aller forcément de soi tant la carence en matière d'annonce de risques d'inondations et le manque d'application de PPRI sont longtemps apparus patents. 


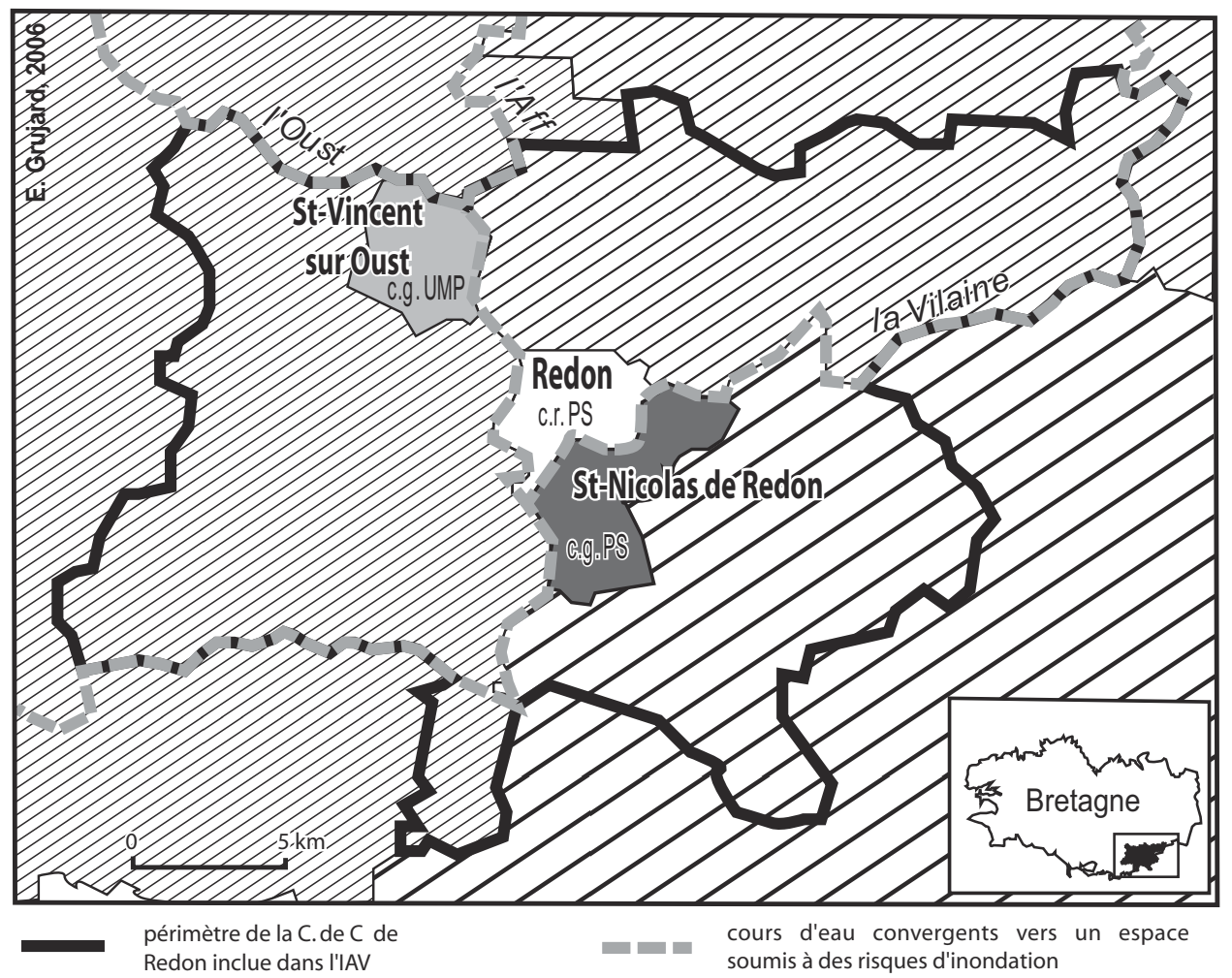

1-perte de la présidence de I'IAV par le Morbihan et l'UMP

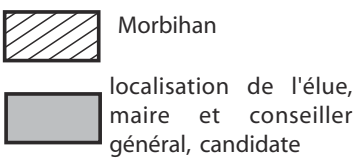

2-conservation de la présidence de la CLE du SAGE par I'Ille et Vilaine mais perte pour l'UMP et gain pour le PS 3-gain de la présidence de l'IAV pour la Loire Atlantique et le PS

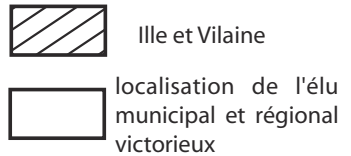

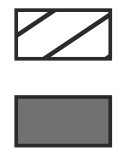

Loire Atlantique

localisation de l'élu conseiller général victorieux

Figure 2 : Évolution géopolitique de la gestion de l'eau dans la communauté de communes du Pays de Redon suite aux élections régionales et cantonales de 2004

Geopolitical evolution of the management of water in the local gouvernement system of the Pays de Redon following the cantonal and regional elections of 2004

\section{Conclusion}

L'étude géopolitique de l'évolution des projets d'aménagements hydrauliques dans le bassin rennais permet d'apporter un éclairage sur les conditions d'une gestion de la ressource en eau soumise à l'influence du poids politique de différents acteurs territoriaux. La gestion de l'eau apparaît ici comme un instrument de pouvoir sur des territoires de nature à générer des rivalités pour l'usage de ces territoires. Pourtant, à la fois l'intrication de ces territoires de l'eau et un certain partage de l'analyse et des représentations des enjeux a finalement tendu à favoriser l'exercice de la concertation entre gestionnaires, en particulier dans le cadre de la procédure d'un SAGE.

Les rivalités en questions ont davantage concerné des enjeux de compétence et de maîtrise d'ouvrage voire de simple influence territoriale laissant le plus souvent un espace ténu pour la pro- 


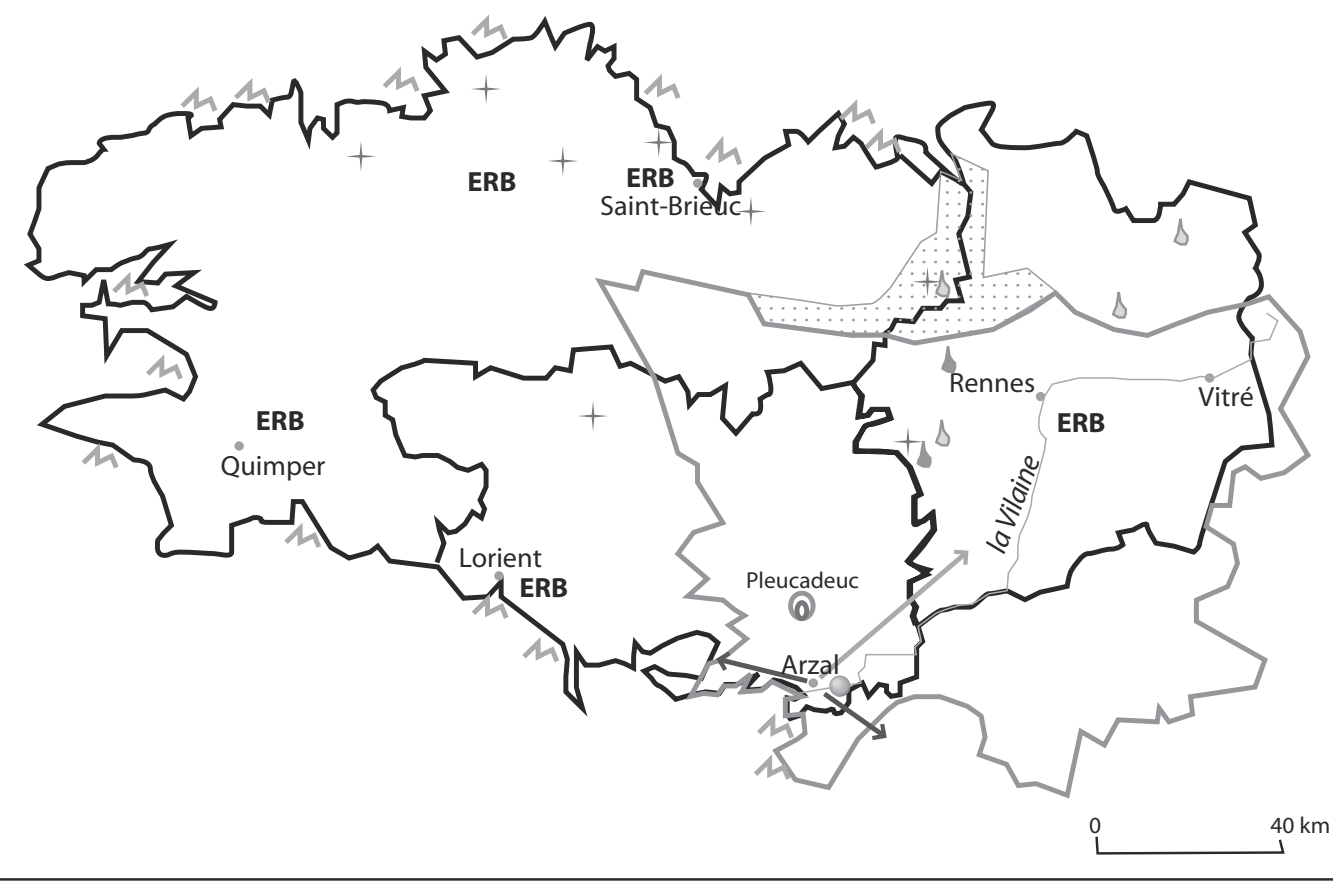

1-des territoires de l'eau rivaux : $\quad$ 2-des capacités d'AEP à conforter :

Rival territories of the water

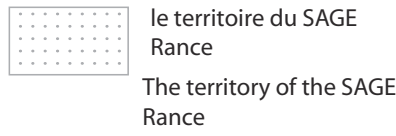

La Roche-Bernard: siège de I'IAV

La Roche-Bernard: seat of IAV

périmètre du SAGE Vilaine

et territoire de I'IAV

Perimeter of the SAGE Vilaine and territory of the IAV

$\longrightarrow$ "ventes" d'eau potable en dehors du bassin de la Vilaine

"Sales" of drinking water outside the basin of Vilaine

(C) localisation du président de I'IAV jusqu'en 2004
Capacities of the drinkable water supply to be consolidated

ressources AEP du SMPBR Resources of the SMPBR

projets inaboutis de création de nouvelles ressources Inaboutis projects of creation of new resources

vers "la grande boucle de solidarité départementale"

Towards the " big buckle of departmental solidarity" 3-un contexte breton très conflictuel :

A very conflicting Breton context

+ manifestations et conflits médiatisés

Demonstrations and mediatized conflicts

ERB implantation d'Eau et rivières de Bretagne Presence of "Eau et rivières de Bretagne"

$M$ risque d'apparition d'algues vertes

Risk of appearance of green seaweeds

Figure 3 : Le contexte géopolique régional du «bassin rennais »

The regional geopolitical context of the "Rennes basin" 
duction d'un discours politique réellement divergent. Au contraire, l'enchaînement de procédures multiples de concertations a permis de faire émerger les conditions d'une gouvernance de l'eau qui semble avoir favorisé une certaine dilution des responsabilités des élus quant aux décisions prises.

Ce qui unit les acteurs de la gestion de l'eau est sans doute plus fort que ce qui les divise. En effet, ces mécanismes d'actions politiques à géométrie variable où des acteurs/décideurs déjà en grande proximité organisationnelle et/ou institutionnelle fonctionnent en réseau (CLE, SMGD, Institut pour l'Aménagement de la Vilaine, services départementaux), plutôt que sur un territoire parfaitement circonscrit, permettent indubitablement une diffusion des risques politiques encourus par des décisions qui se prennent en renvoyant celles-ci moins à un pouvoir local qu'à un faisceau de territoires de gestion de l'eau multiples et moins aisément identifiables.

La Région Bretagne a déployé en vain une attitude très volontariste pour réclamer que la nouvelle loi sur l'eau retienne sa demande d'expérimentation d'un rôle de « chef de file », et regrette que les promesses pourtant faites au précédent exécutif régional ne soient pas tenues par le gouvernement. Ce qui permet d'alimenter cette polémique qui perdure entre les régions et le gouvernement sur les conditions de la décentralisation, cette controverse sur les moyens permettant également sans doute de masquer combien les possibilités d’infléchir la situation restent assez limitées.

Pourtant, au regard de l'ampleur des conflits qui se déroulent en Bretagne entre lobbies économiques et agricoles, entre syndicats professionnels agricoles, entre écologie politique et partis traditionnels, pour ne citer que les plus courants, il est permis de s'interroger quant au réel poids politique du bassin-versant comme territoire de pouvoir. La très vertueuse territorialisation des politiques de l'eau peine à cacher combien cette tentative de construction de solidarités à l'échelle du bassin-versant résiste assez mal à l'épreuve de l'affrontement des divers intérêts économiques, politiques et autres rivalités de territoires de l'eau (fig. 3).

Mais ne sous-estimons pas la capacité de ces multiples protagonistes à se saisir et s'approprier de nouveaux concepts. Le cas de la Ville de Rennes nous le confirme qui, confrontée dans sa délicate relation avec le monde rural à la nécessité de sécuriser l'approvisionnement en eau de son syndicat de gestion dans un contexte de dégradation de la qualité des ressources par la pollution diffuse, a su parfaitement intégrer la notion de développement durable dans la moindre politique publique de reconquête de l'eau afin de préserver l'intégrité de son territoire de l'eau. Ainsi, l'eau et sa gestion font-ils système et s'insèrent parfaitement dans des jeux de relations de pouvoir à plusieurs niveaux de territoires. Ces systèmes d'acteurs, à la fois comme configuration, comme réseaux de pouvoirs et comme jeux d'influence, se caractérisent par des modes d'action et de pouvoir qui se fondent sur des domaines de compétences très divers (expertise, maîtrise d'ouvrage, position dominante sur un marché de l'eau, montage de financements, capacités financières...). La description de ces capacités d'agir spécifiques apportent des éléments essentiels à l'analyse géopolitique par l'identification et l'évaluation des pouvoirs à l'œuvre (Subra, 2007). Si l'on considère le développement durable comme une véritable stratégie pour conquérir ou contrôler l'usage des territoires, il apparaît utile de tenter de comprendre comment les acteurs se saisissent tactiquement de cette doctrine. Ainsi, prendre en compte le rôle des représentations dans la mobilisation des opinions révèle, et constitue comme connaissance à partager, ce que sont les conditions géopolitiques du développement durable appliqué à la gestion de l’eau.

\section{Bibliographie}

Allain (S.), Grujard (E.), Raulet-Croset (N.), 2005. - Décisions et délibérations dans les projets de barrageréservoir vis-à-vis de la régulation politique du domaine de l'eau, Rapport final, MEDD - "Programme Concertation, Décision, Environnement », Paris, ministère de l'Écologie et du Développement Durable, $391 \mathrm{p}$.

Chardronnet (J.), 1980. - Rennes et la Haute Bretagne, Paris, Édition France-Empire, 405 p. 
Gauchon (P.), Tellenne (C.) (dir.), 2005. - Géopolitique du développement durable, Rapport Antheios - septembre 2005, Paris, PUF, 384 p.

Giblin (B.), 2005. - Nowvelles géopolitique des régions françaises, Paris, Fayard, 980 p.

Giblin (B.), 2007. - « Géopolitique et développement durable », dans VEYRET (Y.) (dir.), Le développement durable, Paris, Sedes, p. 269-286.

—, 2003. - « L'eau, une question géopolitique, en France aussi », dans Les Pouvoirs locanx, l'eau, les territoires, Hérodote, $\mathrm{n}^{\circ} 110$, p. 9-28.

GRUjARD (E.), 2006. - Les enjeux géopolitiques de la préservation de la ressource en eau en France, thèse de doctorat, Université Paris 8, 470 p.

—, 2003, « La gestion de l'eau à l'épreuve des territoires », Hérodote, nº 110, p. 47-69.

Le Rhun (P.-Y.), 1988. - Bretagne et Grand Ouest, Morlaix, Skol Vreizh, 130 p.

—, (dir.), 1994. - Géographie et aménagement de la Bretagne, Morlaix, Skol Vreizh, 240 p.

Loyer (B.), 2005. - « Bretagne », dans Giblin (B.) (dir.), Nouvelle géopolitique des régions françaises, Paris, Fayard, p. 276-325.

Martin (C.), 2003. - Mécanismes hydrologiques et hydrochimiques impliqués dans les variations saisonnières des teneurs en nitrate dans les bassins versants agricoles. Approche expérimentale et modélisation, thèse de doctorat, Université de Rennes 1, 267 p.

Menesguen (A.), 2003. - Les « marées vertes » en Bretagne, la responsabilité du nitrate, Direction de l'environnement et de l'aménagement du littoral, IFREMER/Centre de Brest, 11 p.

Nicolazo (J.-L.), Redaud (J.-L.), 2007. - Les agences de l'eau. Quarante ans de politique de l'eau, Paris, Johanet, $376 \mathrm{p}$.

Ollivro (J.), 2000. - La Bretagne en l'an 2000, diagnostics et tendances prospectives, Rennes, PUR, 104 p.

—, 2005a. - Les paradoxes de la Bretagne, Rennes, Apogée, 175 p.

—, 2005b. - Bretagne, 150 ans d'évolution démographique, Rennes, PUR, 368 p.

Petrella (R.) (dir.), 2003. - L'eau Res publica ou marchandise?, Paris, La Dispute, 219 p.

Subra (P.), 2007. - Géopolitique de l'Aménagement du territoire, Paris, Colin, 325 p.

\section{SigLeS UTILISÉS}

AEP : Alimentation en eau potable.

BEP : Bretagne eau pure.

CLE : Commission locale de l'eau.

CGE : Compagnie générale des eaux.

CTE : Contrat territorial d'exploitation.

DCE : Directive cadre européenne sur l'eau.

DDAF : Direction départementale de l'agriculture et des forêts.

DDASS : Direction départementale de l'action sanitaire et sociale.

DDE : Direction départementale de l'équipement.

DIREN : Direction régionale de l'environnement.

DSP : Délégation de service public.

DUP : Déclaration d'utilité publique.

EPCI : Établissement public de coopération intercommunale.

EPTB : Établissement public territorial de bassin.

FNSEA : Fédération nationale des syndicats d'exploitants agricoles.

IAV : Institut d'aménagement de la Vilaine.

IFEN : Institut Français de l'environnement.

IFREMER : Institut français de recherche pour l'exploitation de la mer.

MEDD : Ministère de l'Écologie et du Développement Durable.

PMPOA : Programme de maîtrise des pollutions d'origine agricole. 
PPRI : Plan de prévention des risques d'inondations.

SAFER : société d'aménagement foncier et d'établissement rural.

SAGE : Schéma d'aménagement et de gestion des eaux.

SAUR : Société d'aménagement urbain et rural.

SDAGE : Schéma directeur d'aménagements et de gestion des eaux.

SMGD AEP 35 : Syndicat mixte de gestion du fonds départemental pour le développement de la production d'eau potable en Ille-et-Vilaine.

SMPBR : Syndicat mixte de production du bassin rennais.

SRAEP : Schéma Régional d'Alimentation en Eau Potable.

SYMEVAL : Syndicat mixte des eaux de la Valière.

UDB : Union démocratique bretonne.

UMP : Union pour un mouvement populaire.

Cet article a été reçu le le $1^{\text {er }}$ octobre 2007 et définitivement accepté le 10 mars 2008. 